\title{
Rescate arqueológico. Hotel Pensión Europea. Posadas. Misiones. Argentina
}

\author{
Marianela Biscaldi ${ }^{1}$ y Lorena Salvatelli
}

\begin{abstract}
Resumen
El presente trabajo expone las acciones arqueológicas realizadas en un contexto urbano, mediante un rescate patrimonial. El Departamento de Patrimonio Histórico, Dirección de Urbanismo, Secretaría de Planificación Estratégica y Territorial de la Municipalidad de la ciudad de Posadas (Misiones), solicita al Museo Histórico y Arqueológico “Andrés Guacurarí,, la intervención arqueológica al producirse el hallazgo de una estructura en medio de una obra en construcción, llevada a cabo por la empresa constructora Giovinazzo S.A.

La ciudad de Posadas comienza un proceso de urbanización a principios de siglo XX, por lo cual, el paisaje se transforma, así como la vida de sus habitantes. Este crecimiento implicó la necesidad de tener lugares apropiados para los viajeros, recordemos que es una ciudad de frontera y centro administrativo del entonces territorio nacional de Misiones.

La estructura hallada en pleno casco céntrico de la ciudad, en terrenos que hoy en día se ubican en la intersección de las calles Buenos Aires y Santa Fe, se vinculaba a una cisterna perteneciente al Hotel Pensión Europea.
\end{abstract}

Palabras clave: rescate- intervención arqueológica- contexto urbano- cisterna

\begin{abstract}
The present work exposes the archaeological actions carried out in an urban context, through a patrimonial rescue. The Department of Historic Heritage, Urban Planning Department, Strategic and Territorial Planning Department of the Municipality of the city of Posadas (Misiones), requests the Historical and Archaeological Museum "Andrés Guacurarí", the archaeological intervention when the discovery of a structure in Medium of a work under construction, carried out by the construction company Giovinazzo SA.

The city of Posadas begins a process of urbanization at the beginning of century XX, by which, the landscape is transformed, as well as the life of its inhabitants. This growth implied the need to have appropriate places for travelers, remember that it is a border city and administrative center of the then national territory of Misiones.

The structure found in the center of the city, on land that nowadays is located at the intersection of Buenos Aires and Santa Fe streets, was linked to a cistern belonging to the Hotel Pensión Europea.

Keywords: Rescue - archaeological intervention - urban context - cistern

\footnotetext{
${ }^{1}$ Centro de Estudios de Arqueología Histórica. FHyA. Universidad Nacional de Rosario y Personal externo de Museo Histórico y Arqueológico “Andrés Guacurarí”. marianelabis@yahoo.com.ar

2 Coordinadora Museo Histórico y Arqueológico “Andrés Guacurarí”. Posadas, Misiones; y Centro de Estudios de Arqueología Histórica. FHyA. Universidad Nacional de Rosario. 1salvatelli@gmail.com
} 


\section{Introducción}

En la ciudad de Posadas, a principios del año 2016, fue detectada una estructura en una obra en construcción en un predio céntrico de esta urbe, más precisamente en la intersección de las calles Buenos Aires y Santa Fe. La empresa constructora Giovinazzo S.A se encuentra llevando adelante un emprendimiento inmobiliario, Fideicomiso Rio VII, dirección: Santa Fe 1686; proyecto, dirección y cálculo de estructura Ing. Luis Antonio Giovinazzo Mat. No 553, uso: locales comerciales y vivienda multifamiliar. Expediente No 26981/G/2015; permiso de obra 054/2016.

El arquitecto Darío Avila del Departamento de Patrimonio Histórico, Dirección de Urbanismo, Secretaría de Planificación Estratégica y Territorial de la Municipalidad de Posadas, solicitó la intervención arqueológica al Museo Histórico y Arqueológico "Andrés Guacurarí" de esta ciudad.

\section{Arqueología de rescate y urbana}

La arqueología estudia a través de la cultura material, la vida de sociedades pasadas. Reconstruye la cotidianeidad a través de esos vestigios producidos por el hombre a lo largo del tiempo.

En este sentido, esta intervención arqueológica se produce en el marco de un trabajo de rescate, es decir, salvaguardar el patrimonio que se encuentre en peligro de desaparición parcial o total, ya sean estructuras completas o fragmentadas y todos los materiales asociados a las mismas, en el caso de situaciones particulares, tales como obras en construcción por parte de particulares o entidades públicas. Los tiempos para llevar adelante la labor son acotados e intensos, debido a que se realizan teniendo en cuenta que dichas obras en construcción deberían ser suspendidas hasta tanto no se lleven a cabo las tareas arqueológicas. Sin embargo, las actividades de la obra, generalmente, prosiguen en sectores aledaños que no afectan la excavación arqueológica.

El trabajo arqueológico se realiza respetando los criterios éticos y profesionales para recuperar y preservar el patrimonio; y siguiendo la normativa vigente como la ley Nacional de Protección del Patrimonio Arqueológico y Paleontológico $\mathrm{N}^{\mathrm{o}} 25.743 / 2003$ y su Decreto Reglamentario $\mathrm{N}^{\mathrm{o}}$ 1022/04 ${ }^{1}$, así también lo deja explícito la ley Provincial No 1280 de Patrimonio Cultural/ Decreto Reglamentario No 2530/07.

Para comprender el presente de Posadas, se debe conocer e indagar en su pasado; en sus comienzos como asentamiento jesuítico-guaraní, sus procesos de transformación, su desarrollo durante el siglo XIX, y su constitución como ciudad moderna en el siglo XX.

El proceso de urbanización mayor se generó a principios de siglo XX, se desarrolló inicialmente en torno al puerto, con la Bajada Vieja como camino central hacia el centro de la villa, y siendo el río vía principal de comunicación y comercio, por lo cual, el paisaje cambia, así como la vida de sus habitantes (Areco 1972).

Las transformaciones más notorias que sufrió la ciudad fue el paulatino aumento de su población, y por consiguiente, de su sector económico-productivo. Se estima que hacia el año 1872 el asentamiento urbano contaba con 2000 habitantes, pero al momento de su Federalización ya había ascendido a aproximadamente 4500, es decir, había doblado su población y constantemente llegaban familias a asentarse en sus alrededores (Freaza y Etorena 2010). Según el agrimensor Juan Queirel (1897), informa en su relato "Misiones", que de acuerdo al censo de 1895 la población de Posadas era de 5816 habitantes aproximadamente.

Las recolecciones de datos poblacionales desde 1947 no se discriminan las nacionalidades de los extranjeros, es interesante destacar el paulatino crecimiento de pobladores extranjeros desde finales del s. XIX; dado el contexto histórico que fue desarrollándose en cada momento (revoluciones Europa, Asia y África) y las continuas reconfiguraciones de las formas legales vigentes en aquellos 
tiempos. En este sentido, el constante aumento de población de Posadas, se ve reflejada en la expansión habitacional que modela la topografía citadina (Freaza y Etorena 2010).

Este crecimiento implicó la necesidad de tener lugares apropiados para los viajeros, recordemos que es una ciudad de frontera y centro administrativo del entonces territorio nacional de Misiones ${ }^{2}$. Entre los hospedajes se encontraba el Hotel Pensión Europea, en terrenos que hoy en día se ubican en la intersección de las calles Buenos Aires y Santa Fe, pleno casco céntrico de la ciudad.

\section{Trabajo arqueológico}

El rescate se llevó a cabo entre los días 11 y 23 de abril de 2016, respetando los plazos estipulados en el plan de trabajo presentado a la municipalidad. Se dialogó con el encargado de la Constructora Giovinazzo S.A, Sr. Diego Navaja, y se acordó que se contaría con tres o cuatro operarios para realizar las tareas y poder llevar adelante los objetivos de nuestras labores.

Las áreas a excavar estuvieron delimitadas por las contingencias que se presentaron en el terreno, teniendo en cuenta sobre todo que gran parte de la ocupación más reciente (estacionamiento para autos) ya fue derrumbado, por lo que se planificó una trinchera de excavación en el piso sobre la estructura hallada y una excavación en el sector de la cisterna (posicionamiento geográfico con

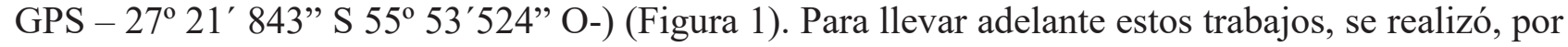
cuestiones de seguridad, el apuntalamiento de la estructura.

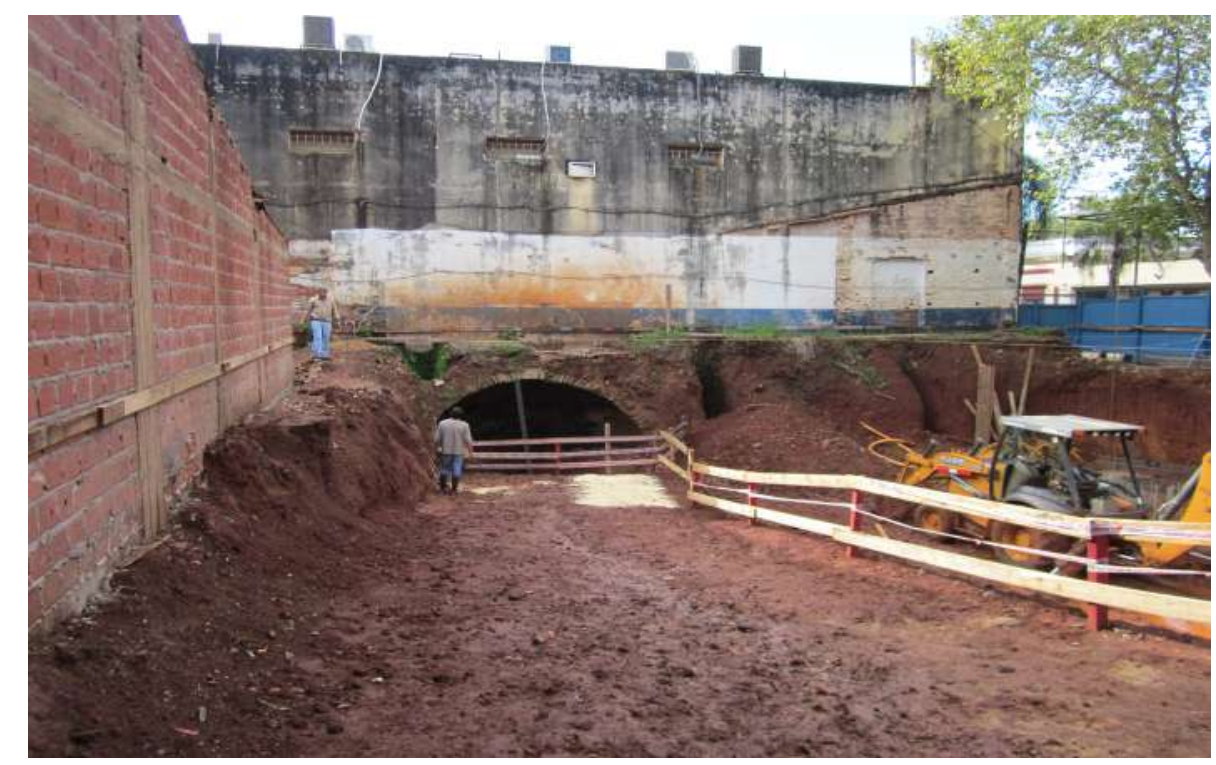

Figura 1. Estructura de Cisterna hallada en obra en construcción. Posadas. Misiones

En primer lugar, se trabajó sobre la Trinchera, las medidas eran de 2,50 mts. x $2 \mathrm{mts}$, se detectaron tres unidades estratigráficas, UE 1 (Unidad Estratigráfica) (Carandini 1997) contrapiso de lo que fue la ocupación más reciente, lavadero de autos (Figura 2). 


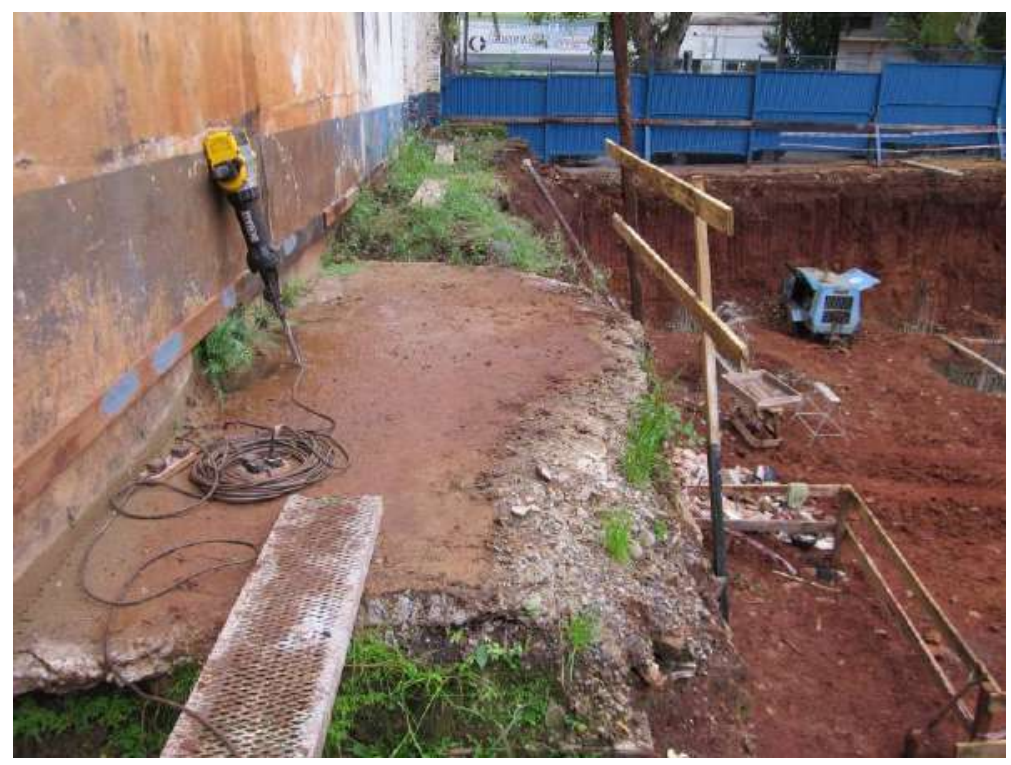

Figura 2.Sector Trinchera Piso sobre Cisterna. UE1

UE 2, a $15 \mathrm{~cm}$. de profundidad, se halló un piso de baldosas, una hilada de ladrillos y una rejilla de hierro fundida. Aquí se observa una división de ladrillos por lo cual se fracciona a la trinchera en A y B. Los hallazgos encontrados fueron: pavimento, rejilla de hierro fundido y ladrillos (Figura 3).

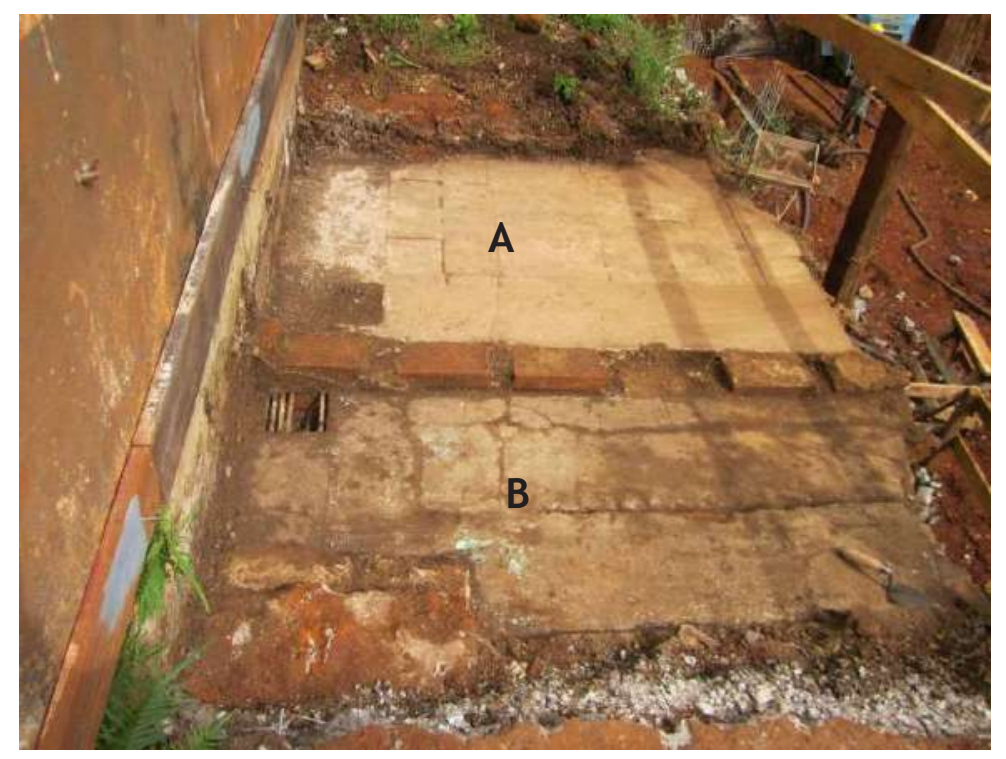

Figura 3. Sector Trinchera Piso sobre Cisterna. UE2

UE 3, a $25 \mathrm{~cm}$ de profundidad; se observó en el sector A un desagüe de ladrillos (albañal) y otro de caño cemento, también se advirtió una especie de receptáculo al final del caño. En el sector B, se detectó un desagüe de ladrillos (albañal) y otro por medio de un caño cerámico. Los restos materiales fueron: ladrillos, fragmentos de loza ${ }^{3}$, fragmentos muy pequeños de óseo de fauna -en el cubículo de la rejilla-. No se extrajo el caño de cemento porque su fabricación es reciente; pero si se liberó el caño 
cerámico, utilizados hasta fines del siglo XIX, que medía 1,39 mt. Allí culmina la excavación en la trinchera (Figura 4).

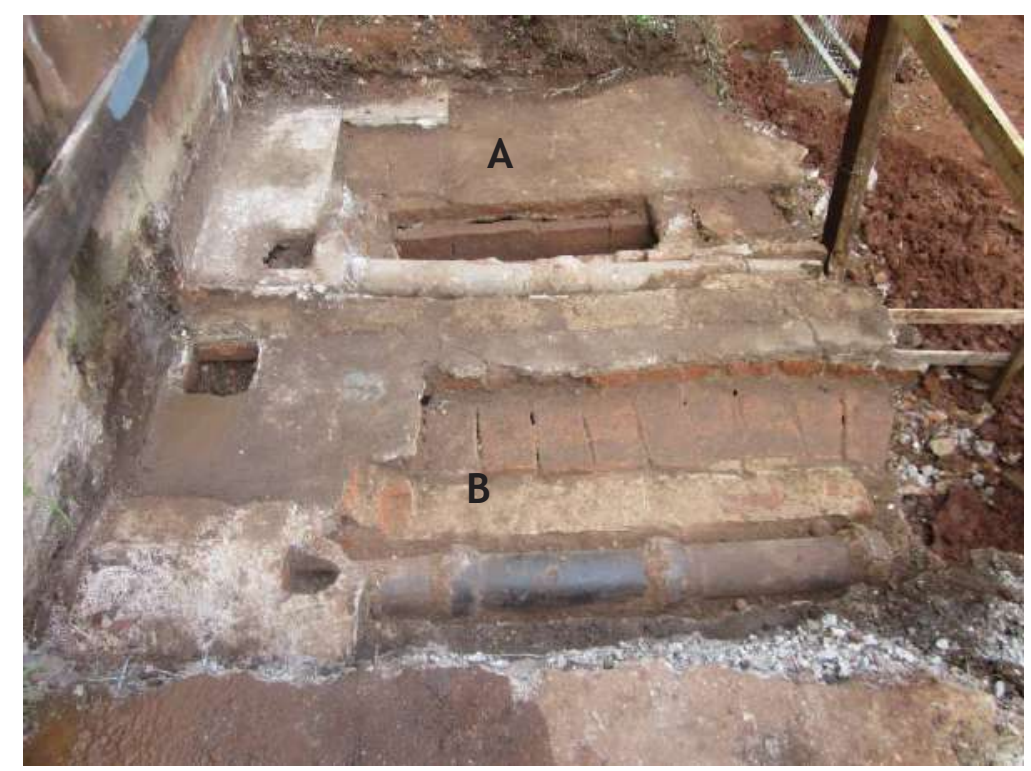

Figura 4. Sector Trinchera Piso sobre Cisterna. UE3

En el perfil que quedaba a la vista, se pudo verificar la profundidad de los diferentes estratos de pisos. Esa profundidad corresponde a $90 \mathrm{~cm}$, y hacia un costado se observó un tirante de madera. Se pudo distinguir una secuencia estratigráfica ${ }^{4}$-desde arriba hacia abajo-: 1) contrapiso de cemento y relleno, 2) pavimento (piso de baldosas con dibujo), 3) ladrillos, relleno, y 4) pavimento (baldosas con ladrillos).

Se continúa con la segunda parte de la excavación y se extrae el material de relleno de la estructura: la cisterna de agua:

(...) era un sistema que almacenaba el agua proveniente de terrazas y patios transportada mediante albañales o caños, el agua se extraía mediante un agujero redondo en la cúpula. Era habitualmente, aunque no siempre, un pozo cilíndrico cubierto, estaba totalmente hecho en mampostería de ladrillo y revocado, con piso de baldosas o ladrillo (Schávelzon, 2010, p. 03).

Se tomaron medidas de lo visible de la cisterna; al comienzo la estructura media: 5,80 mts desde la pared interna de la estructura hacia la barrera de contención; y 3,10 mts desde pared interna hacia el punto medio de la cisterna, donde se tomó el punto de posicionamiento geográfico.

Los operarios retiraron con pala aproximadamente unos $80 \mathrm{~cm}$ de profundidad del relleno. Se intentó pasar ese material por zaranda, pero la consistencia del sedimento no permitió utilizar ese método, por lo que se realizó una revisión manual por cada palada extraída. Tomando en cuenta lo informado por los trabajadores de la obra, el esfuerzo requerido para retirar ese sedimento, el tiempo dispuesto para el rescate; y que se trataba de redepositación de parte del derrumbe, se decidió utilizar la pala mecánica para retirar lo que quedaba y llegar al fondo de la construcción, realizando una revisión por cada extracción. Los hallazgos en este sector fueron: fragmentos de madera, chapa, caño cerámico, ladrillos enteros, clavos, botellas de vidrio, fragmentos de loza, vidrios, pavimento, óseo de fauna, entre otros.

Al llegar al piso de la Cisterna, se limpió y se tomaron las medidas nuevamente para calcular el volumen y la capacidad de almacenamiento de agua. Alto: 5,21 mts; Ancho: 5,45mts (de Este a Oeste) y 5,50mts (de Norte a Sur); Ancho zócalo en el piso de la Cisterna: 0,30mt. A partir de estas medidas 
se calculó que el Volumen de la Cisterna era de: 97,97 m3 y la Capacidad de almacenamiento: 97. 970 lt.

Una tarea anexa fue el relevamiento del inmueble lindero a la obra, que formaba parte de la residencia original del hotel. El estilo arquitectónico de la construcción, poseía características y referencias estéticas provenientes de Italia a fines del siglo XIX, muy utilizadas en la incipiente ciudad.

Esta propiedad muestra un momento de auge del urbanismo de la ciudad, así como la diversidad cultural que caracteriza a esta provincia.

En el predio de la obra se preparó un laboratorio y allí se sistematizaron los restos materiales hallados en el rescate. Se llevó a cabo una clasificación, registro fotográfico y rotulación. Luego de este trabajo, los materiales fueron entregados en custodia al Museo Histórico y Arqueológico "Andrés Guacurarí"

Una vez finalizados las labores de rescate arqueológico, se realizó una jornada de visitas e información destinada a la comunidad en general, denominada "La Historia que Espera". Esto fue posible gracias a la buena predisposición de la empresa constructora y el trabajo coordinado con la municipalidad de Posadas. Este tipo de actividades de extensión del conocimiento científico implica un compromiso y responsabilidad de los profesionales a cargo (poner de manifiesto el uso social de la arqueología), para que la información obtenida del trabajo pueda fortalecer y enriquecer la memoria colectiva y la identidad de la comunidad, ya que sería la forma más adecuada de preservar el patrimonio de los posadeños.

\section{Consideraciones finales}

En el país se han hallado estructuras similares a la relevada en el rescate arqueológico del Sitio Hotel Europeo Posadas. Trabajos como los de Daniel Schávelzon en la provincia de Buenos Aires (Schávelzon, 1999; 2011 y 2013) y Carlos Cerutti en Entre Ríos (Cerutti y Beghetto 2012) -entre otros- demuestran la existencia de Sistemas de almacenamiento de agua (Cisternas) para uso cotidiano, en contextos de incipiente urbanización.

La arqueología urbana de la ciudad de Posadas es escasa, si bien se realizaron trabajos en el casco céntrico, a cargo de la Lic. Ruth A. Poujade, (Trinchera San José en la década de 1980, y Plaza 9 de Julio en la década de 1990 -Poujade 2002-) puede inferirse, a partir del descubrimiento de la estructura de calle Buenos Aires y Santa Fe, que la potencialidad arqueológica es muy grande.

Tal como se mencionó en la planificación del rescate, en las ciudades existe una gran cantidad de problemas metodológicos y técnicos que debemos afrontar en un proyecto que se relaciona con la formación del sitio como lugar arqueológico. El aspecto más interesante es la transformación que sufre la topografía del área urbana desde las primeras épocas de su ocupación ${ }^{5}$; ello genera un gran potencial arqueológico de ese relleno, producto de la reutilización del espacio por las sucesivas generaciones.

En este sentido, el trabajo realizado sienta un precedente más en arqueología urbana y de rescate para la provincia de Misiones, al mismo tiempo que un antecedente de trabajo interdisciplinario -arquitectos, ingenieros, arqueólogos- como también la coordinación de entidades públicas (Municipalidad de Posadas, Museo Histórico y arqueológico “Andrés Guacurarí”) con particulares (Empresa Constructora Giuovinazzo S.A).

La estructura hallada en forma fortuita y relevada durante el rescate arqueológico, correspondería a un tipo de construcción utilizada a principios del siglo XX, para el almacenamiento de agua. Es probable que se haya construido para servir al funcionamiento del Hotel Pensión Europea, ya que, para fines de la década de 1930, comenzaron a funcionar (sobre todo en la provincia de Buenos Aires) los sistemas de agua corriente. Este tipo de información, como la de los materiales asociados, aporta singularidades para la datación relativa del Sitio. 
Si bien los materiales encontrados pueden tener un uso prolongado en el tiempo, pueden dar cuenta del período al que se hace alusión. Existen fragmentos de loza, vidrio (fragmentos de botellas con inscripciones ${ }^{6}$ ) y materiales de construcción (caños cerámicos ${ }^{7}$, ladrillos, tejuelas, rondana de persiana) que indicarían que el espacio estudiado reconstruye la vida cotidiana de la ciudad de Posadas a principios del siglo XX, manifestado a través de los vestigios de lo que fue el Hotel Pensión Europea.

Cabe aclarar que el trabajo de rescate arqueológico es el puntapié inicial para profundizar la temática en una investigación más exhaustiva, que permita tomar medidas para la preservación del Patrimonio Cultural urbano de Posadas.

\section{Notas}

${ }^{1}$ Indicado en el artículo 35. - Cuando los vestigios arqueológicos o paleontológicos se encuentren en terrenos de propiedad privada, la autoridad competente acordará con sus propietarios lo necesario para facilitar el estudio y/o preservación del yacimiento y ARTICULO 36. - El organismo competente podrá, por razones de interés público, disponer la ocupación temporánea de terrenos de propiedad privada donde se localicen lo bienes arqueológicos o restos paleontológicos..."

2 “...Posadas, única población importante, quedara dentro del territorio correntino, el Gdor. Rudecindo Roca solicitó su anexión al Territorio de Misiones, a lo cual accedió el gobierno de Corrientes por Ley del 22/8/1882 (...) el nuevo límite entre Corrientes y Misiones se desplazó hacia el O., por el a. Itaembé, (...) aceptada por la Nación por Ley $N^{\circ} 1.437$ del 30/07/1884, y en su art. $2^{\circ}$ declara capital del Territorio Nacional de Misiones al pueblo de Posadas. Luego de la "Guerra de la Triple Alianza", cobró impulso la explotación de yerbales y extracción forestal en el Alto Paraná, actividades que tenían como base de operaciones los precarios puertos, (...) destacándose Posadas que, como única localidad en formación, tenía su puerto que concentraba todo el tráfico comercial por la histórica Bajada Vieja. Esto generó un gran crecimiento económico que se manifestó en el crecimiento edilicio y el establecimiento de comercios, a lo que agregó luego su carácter de capital administrativa del territorio." (Stefañuk Miguel Ángel. 2009: pp.624)

${ }^{3}$ Material realizado con pasta cerámica fina con una cubierta barnizada, generalmente utilizados para utensilios domésticos, vajilla o materiales sanitarios

4 "estratificación arqueológica: terreno estratificado como consecuencia, en mayor medida, de la acción humana. Se constituye a partir de cambios en las características del material depositado y de mutaciones en las condiciones de deposición; incluye unidades de estratificación creadas mediante deposición y excavación, por ejemplo, estratos y fosas." (Harris Edward C. 1991, p.210)

${ }^{5}$ La cisterna era habitualmente, aunque no siempre, un pozo cilíndrico cubierto con cúpula con el agujero al centro; estaba totalmente hecho en mampostería de ladrillo y revocado, con piso de baldosas o ladrillo" (Schávelzon, 2010 pp.3)

${ }^{6}$ De acuerdo a la publicación de Daniel Schávelzon The Historical Archaeology of Buenos Aires A City at the End of the World (2002:63), los fragmentos hallados podrían ser de Botella Bitter des Basques Archambeaud Freres Bordeaux. Bebida importada a principios del siglo XX.

${ }^{7}$ Caño cerámico vitrificado. Este tipo de conducto de líquidos comienza a utilizarse en el siglo XVIII continuando su uso hasta principios del siglo XX.

\section{Referencias}

ARECO, L. B. (1972). Documentación Histórica 1872-1920. Edición del Bicentenario. Municipalidad de Posadas. Dirección de Cultura y Turismo. Provincia de Misiones. 
CARANDINI, A. (1997). Historia en la Tierra. Manual de excavación arqueológica. Barcelona. España: Ed. Crítica.

CERUTTI, C. N. y SASTRE DE BEGHETTO, A. (2012). Rescate arqueológico en el casco céntrico de Paraná, Entre Ríos: subsuelo del "Plaza Hotel". Recuperado de http://www.ameliasastre. com.ar/2012/11/16/rescate-arqueologico-en-el-casco-centrico-de-parana-entre-rios-subsuelo-del$\%$ E2\%80\%9Cplaza-hotel $\%$ E2\%80\%9D/

FREAZA, J. C. y ETORENA, A. C. (2010). Historia de Posadas. Posadas Misiones, Talleres gráficos EXTRA.

HARRIS, E. C. (1991). Principios de Estratigrafía Arqueológica. Barcelona. España: Ed. Crítica.

POUJADE, R. A. (2002). Arqueología de Rescate (Plaza 9 de Julio -Posadas- Misiones). En XIII Congreso Nacional de Arqueología Argentina. Córdoba, T II. 141 - 154.

QUEIREL, J. (1897). Misiones. Taller Tipográfico de la Penitenciaría Nacional. Buenos Aires.

ROCCHIETTI, A., GERGOLET, S., DE GRANDIS, N. (2008). Arqueología Urbana en la ciudad de Rosario, Provincia de Santa Fe. Argentina. Problemas de arqueología y desarrollo. Revista de la Escuela de Antropología (XIV).

SCHÁVELZON, D. (1999). Arqueología de Buenos Aires. Una ciudad en el fin del mundo. 15801880. Buenos Aires. Argentina: Emecé editores.

SCHÁVELZON, D. (2002). The Historical Archaeology of Buenos Aires A City at the End of the World. Kluwer Academic Publishers New York, Boston, Dordrecht, London, Moscow. Recuperado de http://www.danielschavelzon.com.ar/ebooks/The_Historical_Archaeology_of_Buenos_Aires.pdf

SCHÁVELZON, D. (2013). Lítica Histórica. La piedra de Buenos Aires en los siglos XVI al XX. Usos y tecnologías. Buenos Aires. Argentina: Ed. Aspha.

SCHÁVELZON, D. (2010). Sacando agua y basura en Buenos Aires (siglos XVI al XIX): algunas experiencias arqueológicas. Recuperado de http://www.iaa.fadu.uba.ar/cau/?p=1834

SCHÁVELZON, D. (2011). El aljibe del Colegio Fernando Fader (La Porteña 36). Recuperado de http://www.iaa.fadu.uba.ar/cau/?p=2499

SCHÁVELZON, D. (2014). El aljibe del Asilo Humberto Primo en Villa Devoto: hallazgo y descripción. Recuperado de http://www.iaa.fadu.uba.ar/cau/?p=4212

STEFAÑUK, M.A. (2009). Diccionario Geográfico Toponímico de Misiones. Argentina: Contratiempo Ediciones.

Recibido: $15 / 06 / 2017$

Aceptado: 01/08/2017 